OPEN ACCESS

Edited by:

Haluk Ogmen,

University of Denver, United States

Reviewed by:

Paul Dassonville,

University of Oregon, United States

Takahiro Kawabe,

Nippon Telegraph and Telephone

(Japan), Japan

*Correspondence:

Yujun Pan

yujunpan@ems.hrbmu.edu.cn

Specialty section:

This article was submitted to

Perception Science,

a section of the journal

Frontiers in Neuroscience

Received: 18 January 2017

Accepted: 12 June 2017

Published: 30 June 2017

Citation:

Zhou Y, Li B, Wang G, Zhang M and

Pan Y (2017) Leftward Deviation and

Asymmetric Speed of Egocentric

Judgment between Left and Right

Visual Fields. Front. Neurosci. 11:364.

doi: 10.3389/fnins.2017.00364

\section{Leftward Deviation and Asymmetric Speed of Egocentric Judgment between Left and Right Visual Fields}

\author{
Ying Zhou ${ }^{1}$, Bing $L^{2,3}{ }^{2,}$ Gang Wang ${ }^{1}$, Mingsha Zhang ${ }^{2}$ and Yujun Pan ${ }^{1 *}$ \\ ${ }^{1}$ Department of Neurology, the First Clinical College of Harbin Medical University, Harbin, China, ${ }^{2}$ State Key Laboratory of \\ Cognitive Neuroscience and Learning, Beijing Normal University, Beijing, China, ${ }^{3}$ Institute of Neuroscience, Shanghai \\ Institutes for Biological Sciences, Chinese Academy of Sciences, Shanghai, China
}

The egocentric reference frame is essential for body orientation and spatial localization of external objects. Recent neuroimaging and lesion studies have revealed that the right hemisphere of humans may play a more dominant role in processing egocentric information than the left hemisphere. However, previous studies of egocentric discrimination mainly focused on assessing the accuracy of egocentric judgment, leaving its timing unexplored. In addition, most previous studies never monitored the subjects' eye position during the experiments, so the influence of eye position on egocentric judgment could not be excluded. In the present study, we systematically assessed the processing of egocentric information in healthy human subjects by measuring the location of their visual subjective straight ahead (SSA) and their manual reaction time (RT) during fixation (monitored by eye tracker). In an egocentric discrimination task, subjects were required to judge the position of a visual cue relative to the subjective mid-sagittal plane and respond as quickly as possible. We found that the SSA of all subjects deviated to the left side of the body mid-sagittal plane. In addition, all subjects but one showed the longest RT at the location closest to the SSA; and in population, the RTs in the left visual field (VF) were longer than that in the right VF. These results might be due to the right hemisphere's dominant role in processing egocentric information, and its more prominent representation of the ipsilateral VF than that of the left hemisphere. Keywords: egocentric reference frame, manual reaction time, subjective straight ahead, eye position, asymmetric
perceptions

\section{INTRODUCTION}

Even though the external objects can be represented in multiple reference frames (Goodale and Milner, 1992; Olson, 2003; Burgess, 2006; Milner and Goodale, 2008; Land, 2012; Boccia et al., 2014), the egocentric reference frame is the most fundamental one (Filimon, 2015). The egocentric representation of space is elaborated by the integration of visual, auditory, proprioceptive and vestibular information relative to the eye, head, and torso position of the observer (Andersen et al., 1997). Recent functional magnetic resonance imaging studies have found that the right hemisphere

Abbreviations: PSE, point of subject equality; RT, reaction time; SSA, subjective straight ahead; VF, visual field. 
has more activity than the left hemisphere when healthy righthanded subjects perform egocentric discrimination tasks (Galati et al., 2000, 2001; Neggers et al., 2006; Chen et al., 2012; Saj et al., 2014). The egocentric judgment is frequently tested by measuring the location of subjective straight ahead (SSA), which subjectively separates the egocentric space into left and right halves. Patients with right hemisphere damage more frequently show egocentric neglect of the contralesional hemispace (Beis et al., 2004; Ringman et al., 2004; Becker and Karnath, 2007; Kleinman et al., 2007), as well as an ipsilesional deviation of the SSA (Karnath, 1994; Farne et al., 1998; Ferber and Karnath, 1999; Schindler and Kerkhoff, 2004; Richard et al., 2004a,b, 2005; Saj et al., 2006; Rousseaux et al., 2013). These findings reveal that the processing of egocentric information is asymmetrically distributed between the two hemispheres, with the right hemisphere playing a more dominant role. However, studies of SSA in healthy subjects showed controversial results (Jeannerod and Biguer, 1989; Karnath et al., 1994, 2002; Chokron and Imbert, 1995; McCourt et al., 1997; Vallar et al., 1999; Richard et al., 2004a,b; Saj et al., 2006, 2013; Sumitani et al., 2007; Reinersmann et al., 2012; Rousseaux et al., 2013).

The inconsistent results of SSA in healthy subjects among previous studies might be caused by the employment of different experimental tasks. Two types of tasks were mainly used to measure the location of SSA: the proprioceptive straight ahead pointing task (Heilman et al., 1983) and the visual straight ahead task (Bridgeman and Graziano, 1989). In the former task, subjects pointed to the subjective mid-sagittal plane. In the latter task, subjects either pressed a button or orally reported when a moving visual stimulus reached the subjective mid-sagittal plane (Karnath et al., 1994; Vallar et al., 1999), or adjusted the position of a visual target to the subjective mid-sagittal plane (Bridgeman and Graziano, 1989; Richard et al., 2004b; Saj et al., 2006). In the former task, since the location of SSA was influenced by the pointing hand (left versus right) and the starting position of the hand (Jeannerod and Biguer, 1989; Chokron and Imbert, 1995; McCourt et al., 1997), the results were diverse among the studies. On the other hand, in the visual straight ahead task, only a few studies reported a slight leftward deviation of SSA in healthy right-handed subjects (Sumitani et al., 2007; Reinersmann et al., 2012), while others reported the overlap of SSA with the body mid-sagittal plane (Karnath et al., 1994, 2002; Vallar et al., 1999; Richard et al., 2004a,b; Saj et al., 2006, 2013; Rousseaux et al., 2013). The controversies among previous studies confounded the understanding of the processing of egocentric judgment in the brain.

Another critical issue is that the eye position could influence the judgment of SSA in normal subjects, which might cause the results of previous studies being not consistent. It was reported that the SSA biased either toward (Morgan, 1978) or opposite (Jeannerod and Biguer, 1989; Richard et al., 2005) to the fixation direction. Thus the controversies among previous SSA studies might be due to the influence of the eye position that was not properly monitored. Moreover, previous studies mainly focused on assessing the accuracy of egocentric discrimination, but the timing of egocentric judgment was unexplored.
To address these questions, in the present study, we measured the location of visual SSA and manual reaction time (RT) of healthy human subjects. Our egocentric discrimination task required subjects to keep fixation and press a key as quickly as possible to respond to the egocentric location of a visual cue. We found that the SSA of all subjects deviated to the left side of the body mid-sagittal plane. In addition, all subjects but one showed the longest RT at the location closest to the SSA; and in population, the RTs in the left visual field (VF) were longer than that in the right VF. Thus, our SSA and RT data demonstrate that the right hemisphere of healthy human subjects plays a more dominant role in processing egocentric information. Such results are consistent with the fact that hemispatial neglect more frequently occurs in patients with right hemisphere damage.

\section{MATERIALS AND METHODS}

\section{Subjects}

Sixteen healthy human subjects (age 22-29 years old, 7 males, 9 females) participated in this study. They were all right-handed subjects with normal or corrected to normal vision. We measured the eye dominance of each subject by 3 repeats of the "Holein-card" test (Miles, 1930), and the results were consistent for each individual subject. Among the 16 subjects, 5 subjects were left-eye dominant and 11 subjects were right-eye dominant. All subjects were naive to the experimental purpose. At the early stage of this study, 10 subjects (left-eye dominant: 5, righteye dominant: 5) were recruited in the First Clinical College of Harbin Medical University. Since one subject had difficulty to keep fixation (the fixation break rate $>20 \%$ ), data from this subject were excluded from further analysis. At the late stage of this study, the other 6 subjects (right-eye dominant: 6) were recruited in Beijing Normal University. The protocol of this study followed the ethical guidelines of the Declaration of Helsinki and was approved by the Institutional Review Board of the First Clinical College of Harbin Medical University and Beijing Normal University. All subjects gave written informed consent before participating in this study and received financial compensation for their participation.

\section{Apparatus}

To eliminate the possibility that the surrounding objects might serve as the allocentric referees, all experiments were conducted in a dark room. The computer screen was placed $57 \mathrm{~cm}$ in front of the subjects' eyes. A chin rest restricted any head movement of subjects. When setting up the experimental system, we carefully measured the distance between the chin rest and the screen to ensure that: the chin rest and the screen were parallel in both horizontal and vertical dimensions, and the vertical middle line of the chin rest was aligned with the vertical middle line of the screen. Thus, we did our best to ensure that the screen was centered and oriented parallel to the plane of the chin rest. In addition, we marked the midpoint of the chin rest and instructed the subjects to put their chins on this point before collecting data in each session. And the subjects were instructed to keep the same posture throughout each session. 
At the early stage of this study, a computer keyboard was positioned in front of the subjects, with the up and down keys aligned with their body mid-sagittal plane. The left key and right key were positioned with equal distance from the body midsagittal plane. At the late stage of this study, to exclude the possibility that the leftward deviation of keyboard might cause the deviation of SSA, the keyboard was covered by two black boxes with the same size, only leaving the up, down, left and right keys uncovered. In addition, the up and down keys were aligned with the body mid-sagittal plane.

All visual stimuli were presented on a 21 -inch CRT monitor $($ SUN X7149A, $1280 \times 960$ pixels, $100 \mathrm{~Hz}$ vertical refresh rate) and the luminance of the stimuli was measured with a photometer (LS-110; Konica Minolta). Before starting the experiments, we calibrated and linearized the screen with the photometer. We also presented a circle with a $15^{\circ}$ radius that was centered at the center of the screen. When we measured the distances from multiple points on the circle to the center of the screen, we found that the distances were all $15^{\circ}$. Thus, there was no spatial distortion on the screen display.

We monitored and collected the eye position signal at a sample rate of $1 \mathrm{kHz}$ by an infrared camera eye tracking system (EyeLink 1000 Desktop Mount; SR Research). We used MATLAB (version 2012a; The MathWorks) with Psychtoolbox (PTB-3; Brainard and Pelli, 2015) to control the presentation of the visual stimuli and collect the subjects' RT data.

\section{Behavioral Tasks \\ Egocentric Discrimination Task}

Egocentric discrimination task (Main task, Figure 1A). The trial began with a red fixation point appearing on the screen with black background, located $9^{\circ}$ below the center of the screen. The subjects needed to look at this fixation point within $500 \mathrm{~ms}$ and keep fixation within an invisible circular window (radius: $3^{\circ}$ ) until the end of the trial. If the eye position moved out of the fixation window during the fixation period, the fixation point would turn green and the trial would be terminated. After a random period of $600-1600 \mathrm{~ms}$, a green circle (visual cue) would appear on the screen (radius: $0.5^{\circ}$, luminance: $0.05 \mathrm{~cd} / \mathrm{m}^{2}$ ) for $200 \mathrm{~ms}$. Subjects needed to judge whether the green circle was in the left or right side of the subjective mid-sagittal plane and press the left or right key accordingly (using the index of left or right hand, respectively) as quickly as possible.

At the early stage of this study, there were 12 possible locations in which the green circle randomly appeared at one of them. All 12 positions were $9^{\circ}$ above the horizontal meridian of the screen in the Y-axis, and with $0.5,1.5,3.5,6.5,10.5$, and $15.5^{\circ}$ to the left and right in the $\mathrm{X}$-axis (Figure 1B). During the experiments, the subjects performed 3 sessions per day for 3 days. Each session contained 120 trials. At the late stage of this study, there were only 6 possible locations (left and right $0.5,1.5$, and $3.5^{\circ}$ ) because the objective of the late stage was to assess the influence of eye position on spatial judgment. The inconsistency of the judgment to the same visual cue was high only at the center locations. During the experiments, the subjects performed 3 sessions per day for 2 days. Each session contained 120 trials.
Considering the possibility that the fixation point might serve as an allocentric cue to facilitate the egocentric judgment of the visual cue, we put the fixation point on the vertical meridian with $9^{\circ}$ below the horizontal meridian, and put the visual cue with $9^{\circ}$ above the horizontal meridian. Thus, when the visual cue appeared close to the vertical meridian, it was difficult for the subjects to judge the egocentric location of the visual cue based on its position relative to the fixation point.

\section{Single Hand Task}

Single hand task (Control task, Figure 1C). The sequence and temporal feature of this task were the same as the main task, with the only difference being that the green circle appeared only at one location: $9^{\circ}$ above the center of the screen. In a given session, subjects were instructed to always press the same key (either left or right) as soon as the green circle appeared. In this task, subjects didn't need to make a judgment about the egocentric location of the visual cue. During the experiments, subjects performed 2 sessions daily (1 session with left key press and 1 session with right key press) at the beginning and the end of the experiment, respectively. Each session was composed of 30 trials. The sequence of left key press and right key press was randomized. This task was only performed at the early stage of this study.

\section{Data Analysis}

In the main task, we excluded fixation break trials (early stage: $4.60 \%, 447$ out of 9,720 trials; late stage: $4.35 \%, 188$ out of 4,320 trials) and no response trials (early stage: $0.01 \%, 1$ out of 9,720 trials; late stage: $0.14 \%$, 6 out of 4,320 trials) from data analysis. In the control task, we first excluded fixation break trials $(4.42 \%$, 138 out of 3,120 trials). Then trials with RT differing more than 3 standard deviations from the mean RT of each day (in total, $1.48 \%, 44$ out of 2,982 trials) were also excluded. The number of no response trials was zero.

\section{Point of Subject Equality (PSE) Calculation}

For each subject, the manual response (e.g., the percentage of pressing right key) at each visual cue location was calculated. A cumulative normal distribution function was used to fit the response percentage data to estimate the psychometric function. And then we defined the point of subject equality (PSE) of each psychometric curve as the point (location in horizontal dimension) at which the percentage of leftward and rightward response were equal (50\%). The PSE was regarded as the location of the visual SSA of every subject. Negative value indicated a leftward deviation whereas positive value indicated a rightward deviation from the body mid-sagittal plane.

\section{RT Calculation}

We intended to explore the egocentric judgment time by measuring the manual RT of two hands. However, considering the RTs between two hands often differ in humans (intrinsic RT difference) (Boulinguez et al., 2001), we should first exclude the influence of the intrinsic RT difference. Thus we calculated the intrinsic RT difference in the control task (single hand task, Figure 1C) by subtracting the mean RTs of the right hand from 
A

Egocentric discrimination task

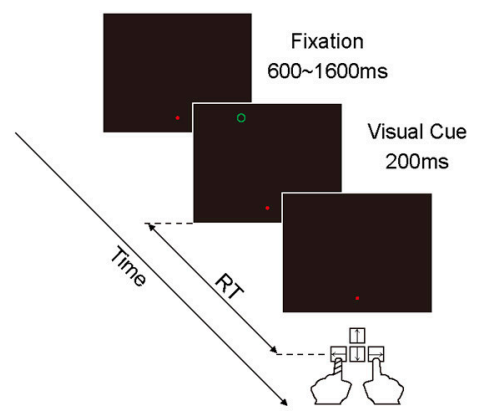

B

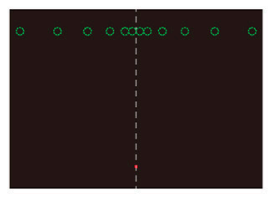

C

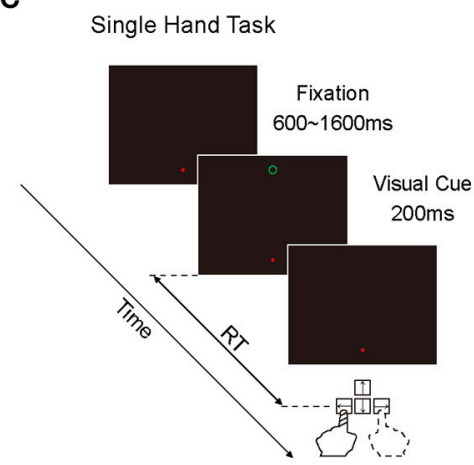

FIGURE 1 | Illustration of the two behavioral tasks. (A) The diagram of egocentric discrimination task. (B) The illustration of the possible egocentric locations of the visual cue. (C) The diagram of single hand task.

the mean RTs of the left hand. Then we calculated the postadjusted RTs in the main task by subtracting the intrinsic RT difference from the RTs of the left hand. The RT data presented in this paper were post-adjusted. We also excluded outliers in which the post-adjusted RT differed more than 3 standard deviations from the mean post-adjusted RT of each day (in total, 1.24\%, 115 out of 9,272 trials).

\section{RESULTS}

At the early stage of this study, 9 subjects performed both the main task (12 visual cue locations) and the control task. The RTs of each individual subject in the control task were relatively consistent across the experimental days, as is shown in Table 1. The results of each individual subject's judgment at each visual cue location in the main task are presented in Table 2. Unsurprisingly, the inconsistent judgment (same visual cue location ended with opposite judgments) occurred more frequently when the visual cue was close to the body mid-sagittal plane. Notably, the frequency of inconsistent judgment was not symmetric between the two most center locations $\left(-0.5^{\circ}\right.$ versus $\left.0.5^{\circ}\right)$, but with significantly higher rate at $-0.5^{\circ}(p=4.1135 \mathrm{e}-$ 05 , Wilcoxon test). The greater rate of inconsistent judgment at $-0.5^{\circ}$ indicated that, compared with $0.5^{\circ}$, it was more difficult to judge the egocentric location of the visual cue at $-0.5^{\circ}$.

\section{The PSE of the Psychometric Curve of Each Subject Deviated to the Left Side of the Body Mid-Sagittal Plane}

To illustrate the subjects' egocentric judgment more clearly, we analyzed the percentage of rightward judgment at each visual cue location. To be noticed, the percentage of leftward judgment was a mirror image of the percentage of rightward judgment (100-the percentage of rightward judgment). The percentages of rightward judgment at each visual cue location in the main task are shown in Figure 2A. Data of each individual subject (same colored triangles) were fitted with a cumulative normal distribution function, shown as the same colored thin curve. The averaged data were denoted as black triangles and thick curve. As expected, the percentages of rightward judgment at the 3 most peripheral locations in the left VF $\left(-15.5,-10.5\right.$, and $\left.-6.5^{\circ}\right)$ were all $0 \%$ and those at the 3 most peripheral locations in the right VF $\left(15.5,10.5\right.$, and $\left.6.5^{\circ}\right)$ were all $100 \%$ among the subjects. When the visual cue got closer to the body mid-sagittal plane (from -3.5 to $3.5^{\circ}$ ), the percentage of rightward judgment gradually increased in the left VF, and it gradually decreased in the right VF (Figure 2B). Such results indicated that, when the visual cue was close to the body mid-sagittal plane, the egocentric judgment to the same location was ended with opposite outputs (leftward versus rightward, Table 2). Strikingly, the fitted psychometric curves of all subjects shifted to the left side of the body midsagittal plane, and the population point of subject equality (PSE) was at $-0.31^{\circ} \pm 0.16^{\circ}$ (mean $\pm \mathrm{SD}$ ). Compared with the body mid-sagittal plane, the leftward deviation of SSA was significant ( $p=4.1135 \mathrm{e}-05$, Wilcoxon test). These results showed that the visual SSA of all subjects deviated to the left side of the body mid-sagittal plane, regardless of the eye dominance.

\section{The RTs Were Longer When the Visual Cue Was Closer to the SSA Rather Than the Body Mid-Sagittal Plane}

We assessed the time of egocentric judgment by calculating the post-adjusted RTs of each individual subject in the main task (Figure 3A). As expected, the RTs gradually prolonged as the visual cue got closer to the body mid-sagittal plane. Notably, the RTs were asymmetric between left and right VFs: RTs were longer in the left VF than in the right VF. Two-way ANOVA showed that both eccentricity $\left[F_{(5,96)}=10.92, p=2.4673 \mathrm{e}-08, \eta_{\mathrm{p}}{ }^{2}=\right.$ $0.36]$ and laterality $\left[F_{(1,96)}=4.12, p=0.045, \eta_{\mathrm{p}}{ }^{2}=0.04\right]$ of the visual cue had significant influence on the RTs. But there was no significant interaction effect between the two factors $\left[F_{(5,96)}=\right.$ $\left.0.32, p=0.901, \eta_{\mathrm{p}}{ }^{2}=0.02\right]$. Moreover, RTs of all subjects but one were longest when the visual cue was closest to the SSA. For instance, the population RT was longest when the visual cue was at $-0.5^{\circ}$ (Figure 3B), consistent with the fact that the mean SSA was located at $-0.31^{\circ}$ (Figure 2B).

We further quantitatively analyzed the RT difference (left VF-right VF) between each mirror location for each individual 
TABLE 1 | Mean RTs of the left and right hand and the intrinsic RT difference between the two hands (left hand-right hand) of individual subject in the control task (unlike the other subjects, subject WG performed the main task with 180 trials per session and completed the experiments within 2 days).

\begin{tabular}{|c|c|c|c|c|c|c|c|c|c|c|c|c|}
\hline \multirow[t]{2}{*}{ Subject } & \multicolumn{4}{|c|}{ Mean RTs of left hand (ms) } & \multicolumn{4}{|c|}{ Mean RTs of right hand (ms) } & \multicolumn{4}{|c|}{$\begin{array}{l}\text { Intrinsic RT difference } \\
\text { between two hands (ms) }\end{array}$} \\
\hline & Day 1 & Day 2 & Day 3 & Mean & Day 1 & Day 2 & Day 3 & Mean & Day 1 & Day 2 & Day 3 & Mean \\
\hline CJL & 425.85 & 397.27 & 364.77 & 395.96 & 457.08 & 400.37 & 378.85 & 412.10 & -31.22 & -3.11 & -14.08 & -16.14 \\
\hline DYC & 410.44 & 397.13 & 342.86 & 383.48 & 379.89 & 354.50 & 328.90 & 354.43 & 30.55 & 42.63 & 13.96 & 29.05 \\
\hline GZ & 318.40 & 305.40 & 292.78 & 305.53 & 315.93 & 301.27 & 291.05 & 302.75 & 2.47 & 4.13 & 1.73 & 2.78 \\
\hline LMJ & 310.28 & 307.05 & 293.91 & 303.75 & 294.89 & 284.74 & 293.85 & 291.16 & 15.39 & 22.31 & 0.05 & 12.58 \\
\hline LYH & 481.99 & 437.30 & 413.16 & 444.15 & 441.94 & 433.73 & 443.68 & 439.79 & 40.05 & 3.56 & -30.52 & 4.37 \\
\hline QHL & 348.12 & 331.61 & 305.50 & 328.41 & 358.75 & 319.85 & 303.08 & 327.22 & -10.63 & 11.76 & 2.43 & 1.18 \\
\hline QSH & 362.37 & 354.65 & 349.29 & 355.44 & 363.84 & 381.07 & 344.39 & 363.10 & -1.47 & -26.41 & 4.90 & -7.66 \\
\hline WG & 362.71 & 338.14 & - & 350.43 & 373.65 & 349.32 & - & 361.48 & -10.94 & -11.17 & - & -11.06 \\
\hline $\mathrm{ZHH}$ & 333.26 & 326.13 & 320.14 & 326.51 & 309.98 & 306.88 & 311.01 & 309.29 & 23.28 & 19.25 & 9.13 & 17.22 \\
\hline
\end{tabular}

TABLE 2 | The egocentric judgments of individual subject in the main task.

\begin{tabular}{|c|c|c|c|c|c|c|c|c|c|c|c|c|}
\hline \multirow[t]{3}{*}{ Subject } & \multicolumn{12}{|c|}{ Location of visual cue } \\
\hline & \multicolumn{6}{|c|}{ Left VF } & \multicolumn{6}{|c|}{ Right VF } \\
\hline & $-15.5^{\circ}$ & $-10.5^{\circ}$ & $-6.5^{\circ}$ & $-3.5^{\circ}$ & $-1.5^{\circ}$ & $-0.5^{\circ}$ & $0.5^{\circ}$ & $1.5^{\circ}$ & $3.5^{\circ}$ & $6.5^{\circ}$ & $10.5^{\circ}$ & $15.5^{\circ}$ \\
\hline \multicolumn{13}{|c|}{ EARLY STAGE } \\
\hline CJL & $80 / 0$ & $86 / 0$ & $81 / 0$ & $81 / 0$ & $74 / 4$ & $37 / 44$ & $79 / 6$ & $82 / 2$ & $80 / 0$ & $83 / 0$ & $82 / 0$ & $86 / 0$ \\
\hline DYC & $87 / 0$ & $85 / 0$ & $85 / 0$ & $84 / 0$ & $81 / 5$ & $50 / 31$ & $68 / 14$ & $84 / 2$ & $83 / 0$ & $88 / 0$ & $83 / 0$ & $87 / 0$ \\
\hline$G Z$ & $89 / 0$ & $88 / 0$ & $89 / 0$ & $86 / 1$ & $81 / 6$ & $54 / 34$ & $72 / 16$ & $87 / 0$ & $86 / 1$ & $89 / 0$ & $88 / 0$ & $88 / 0$ \\
\hline LMJ & $79 / 0$ & $95 / 0$ & $92 / 0$ & $92 / 0$ & $82 / 11$ & $51 / 37$ & $71 / \mathbf{2 0}$ & $87 / 1$ & $91 / 1$ & $79 / 0$ & $79 / 0$ & $78 / 0$ \\
\hline LYH & $88 / 0$ & $85 / 0$ & $84 / 0$ & $88 / 0$ & $80 / 6$ & $55 / 33$ & $81 / 7$ & $85 / 1$ & $87 / 1$ & $84 / 0$ & $82 / 0$ & $83 / 0$ \\
\hline QHL & $86 / 0$ & $81 / 0$ & $86 / 0$ & $88 / 0$ & 73/11 & $37 / 48$ & $68 / 16$ & $88 / 2$ & $88 / 0$ & $87 / 0$ & $86 / 0$ & $87 / 0$ \\
\hline QSH & $89 / 0$ & $89 / 0$ & $88 / 0$ & $87 / 1$ & $89 / 1$ & $57 / \mathbf{3 1}$ & $66 / 19$ & $84 / 4$ & $90 / 0$ & $90 / 0$ & $89 / 0$ & $88 / 0$ \\
\hline WG & $84 / 0$ & $88 / 0$ & $88 / 0$ & $90 / 0$ & $88 / 1$ & $54 / 26$ & $86 / 3$ & $88 / 0$ & $85 / 0$ & $84 / 0$ & $86 / 0$ & $83 / 0$ \\
\hline $\mathrm{ZHH}$ & $83 / 0$ & $84 / 0$ & $84 / 0$ & $83 / 0$ & $81 / 1$ & $63 / 22$ & $70 / 15$ & $83 / 0$ & $84 / 0$ & $86 / 0$ & $83 / 0$ & $87 / 0$ \\
\hline Total & $765 / 0$ & $781 / 0$ & 777/0 & $779 / 2$ & $729 / 46$ & 458/306 & $661 / 116$ & $768 / 12$ & $774 / 3$ & $770 / 0$ & $758 / 0$ & $767 / 0$ \\
\hline \multicolumn{13}{|c|}{ LATE STAGE } \\
\hline DY & - & - & - & $117 / 2$ & $120 / 0$ & $84 / 36$ & $111 / 9$ & $118 / 2$ & $118 / 0$ & - & - & - \\
\hline JFF & - & - & - & $113 / 0$ & $108 / 4$ & $87 / 26$ & 100/11 & $107 / 0$ & $118 / 0$ & - & - & - \\
\hline LM & - & - & - & $110 / 0$ & $110 / 2$ & $73 / 33$ & $105 / 6$ & $116 / 0$ & 109/0 & - & - & - \\
\hline$X F$ & - & - & - & $109 / 2$ & $110 / 0$ & $87 / 27$ & $95 / 20$ & $114 / \mathbf{1}$ & $112 / 0$ & - & - & - \\
\hline YL & - & - & - & $116 / 0$ & $114 / 2$ & $71 / 45$ & 104/13 & $119 / 0$ & $117 / \mathbf{1}$ & - & - & - \\
\hline ZZY & - & - & - & $117 / 0$ & $117 / 0$ & 95/23 & $98 / 11$ & $116 / 3$ & $111 / 0$ & - & - & - \\
\hline Total & - & - & - & $682 / 4$ & $679 / 8$ & 497/190 & $613 / 70$ & $690 / 6$ & $685 / 1$ & - & - & - \\
\hline
\end{tabular}

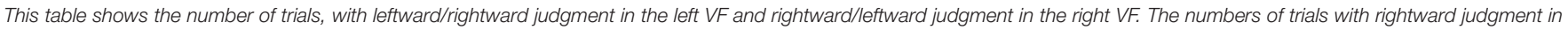
the left VF and leftward judgment in the right VF are highlighted in bold.

subject. The results of the subtracted RTs of each subject are presented with different colored triangles in Figure 4. The solid symbols denote that the RT difference between left and right VFs reached the statistically significant level $(p<0.05$, Wilcoxon test), whereas the dashed symbols do not. As shown, the RT difference between the 2 most center locations $\left(-0.5^{\circ}\right.$ versus $\left.0.5^{\circ}\right)$ was greatest, with the RT difference of 6 out of 9 subjects reaching the statistically significant level. The RT difference gradually decreased as the eccentricity of visual cue increased. Eventually, at the peripheral locations, the RT difference of several subjects reversed.
The longer RT indicated that longer time was needed to judge the egocentric location of the visual cue. Our RT data showed that the most difficult egocentric judgment was around the SSA, rather than the body mid-sagittal plane.

\section{The Leftward Deviation of SSA Was Not Due To the Influence of Eye Position}

At the late stage of this study, 6 subjects performed the main task with only 6 visual cue locations. The objective of the late stage was to assess the influence of eye position on the spatial judgment to 


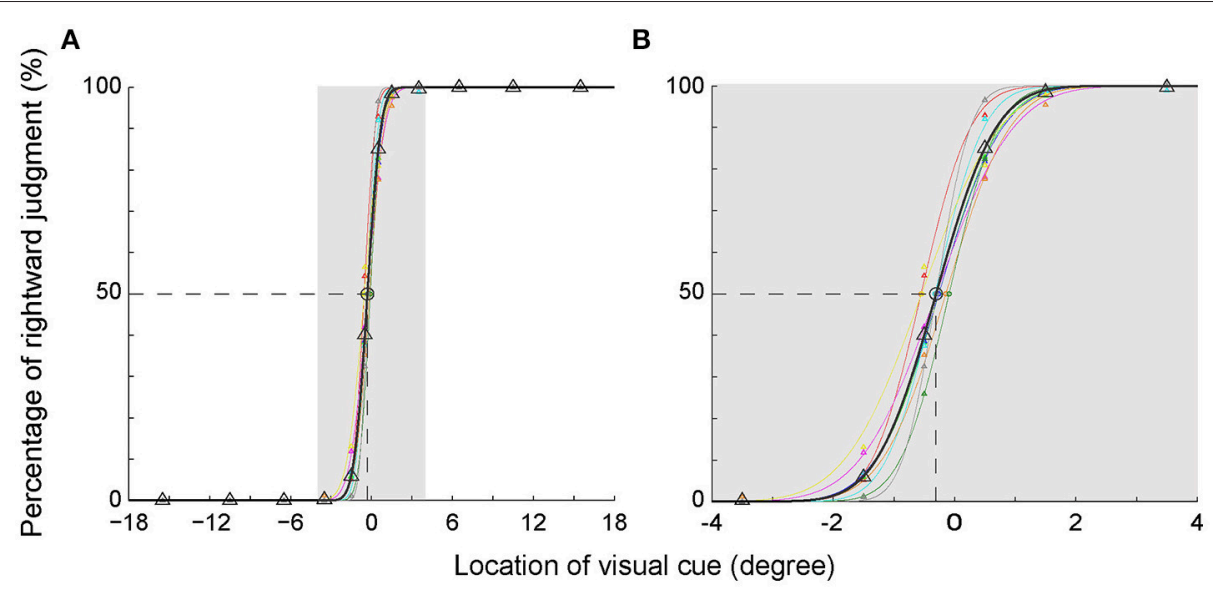

FIGURE 2 | Percentage of the rightward judgment as a function of the egocentric location of the visual cue. (A) The psychometric curve of each individual subject (different colors) and the averaged psychometric curve of population data (black). (B) The enlarged graph of gray area in panel A for clearer vision. The PSEs of all subjects shifted to the left side of the body mid-sagittal plane, with a population PSE: $-0.31^{\circ} \pm 0.16^{\circ}$ (mean \pm SD).

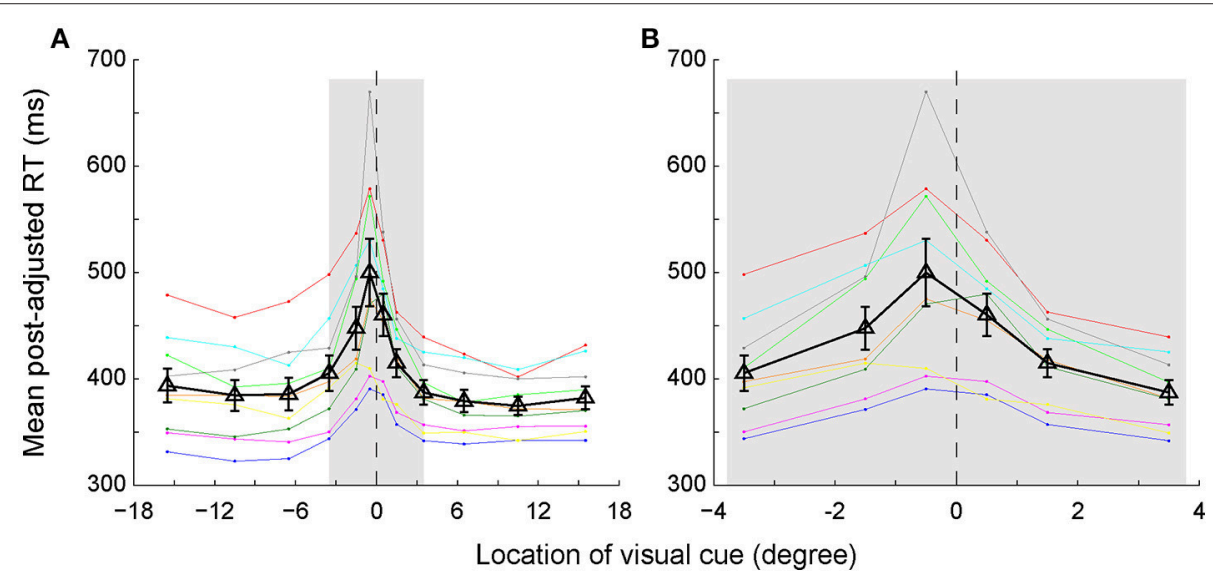

FIGURE 3 | The post-adjusted RTs distribution as a function of the egocentric location of the visual cue. (A) Same colored dots connected with the same colored lines represent one individual subject's RTs. Black triangles connected with black lines represent the averaged RTs of all subjects. The vertical bars represent the standard errors. (B) The enlarged graph of gray area in panel A for clearer vision.

a visual cue. We compared the eye position (averaged from visual cue onset to $250 \mathrm{~ms}$ later) between trials where subjects made two opposite judgments to the same visual cue.

First, the fitted psychometric curves of the rightward judgment percentages of 6 subjects shifted to the left side of the body mid-sagittal plane. The population PSE was at $-0.18^{\circ} \pm$ $0.10^{\circ}$ (mean $\pm \mathrm{SD}$ ) (Figure 5A). Compared with the body midsagittal plane, the leftward deviation of SSA was significant $(p=$ 0.002 , Wilcoxon test), which was consistent with the results of 9 subjects at the early stage.

The location of $-0.5^{\circ}$ was nearest to the location of SSA, thus the inconsistency of judgment (leftward versus rightward) was highest. We compared the eye positions between two groups of trials at $-0.5^{\circ}$. We found that the distribution of the eye position was centered at the fixation point, regardless of the results of spatial judgment (Figures 5B,C). Furthermore, the horizontal eye positions between the two groups of trials were not significantly different both in the individual subject level (except one subject) and in the population level (Figure 5D, $p=0.496$, Wilcoxon test). These results indicated that in the present study, the leftward deviation of SSA was not due to the influence of eye position.

\section{DISCUSSION}

In the present study, we assessed the egocentric judgment of healthy subjects by measuring the location of SSA and manual RT. To exclude the influence of eye position on egocentric judgment, we asked the subjects to keep fixation and monitored the eye position by an infrared camera eye tracking system during the experiments. We found that: (1) the SSA of the subjects all deviated to the left side of the body mid-sagittal plane; (2) the RTs of all subjects but one were longest when the visual cue was nearest to the location 
of SSA, with the RTs in the left VF being longer than that in the right VF; (3) there was no significant difference of horizontal eye positions between the trials with opposite spatial judgments.

\section{The Possible Interpretation of Our Results}

The anatomical and physiological features of visual system of primates might explain our results-the leftward deviation of SSA and longer RT in the left VF. Previous studies have revealed that, while the lower visual areas in one hemisphere strictly process the visual information from contralateral VF

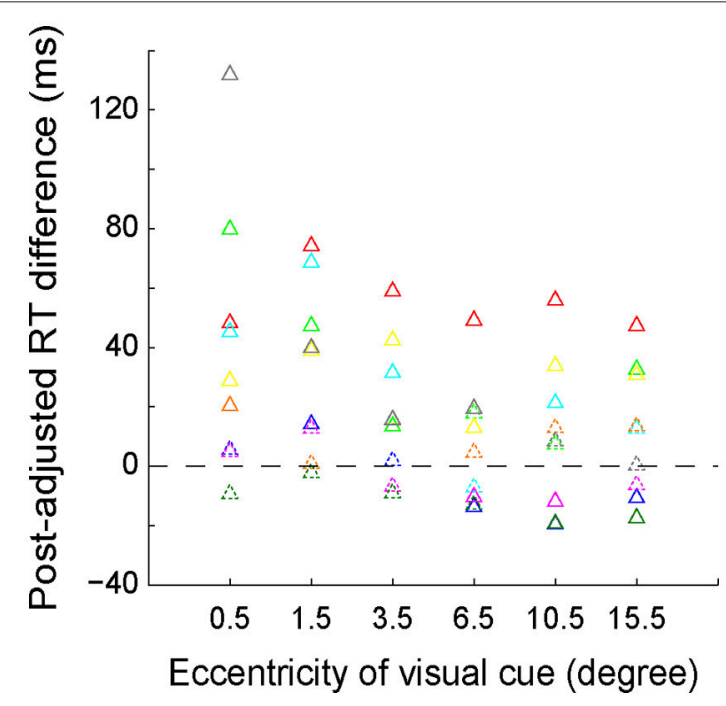

FIGURE 4 | The distribution of post-adjusted RT difference (left VF-right VF) between the 6 mirror locations of the visual cue. The same colored triangles represent the data of one individual subject. Solid symbols indicate that the RT difference reached the statistically significant level ( $\rho<0.05$, Wilcoxon test), whereas the dashed symbols do not.
(Tootell et al., 1982), the higher visual cortices process the visual information from both contralateral and ipsilateral VFs (Gross et al., 1969, 1972; Andersen et al., 1990; Raiguel et al., 1997; Ben Hamed et al., 2001). One reasonable explanation for our results is that the right hemisphere receives and processes more visual information from the ipsilateral VF than the left hemisphere does (Sheremata et al., 2010; Zhou et al., 2012; Sheremata and Silver, 2015). Therefore, compared with the left VF, the right VF might be overestimated, so that the SSA deviated to the left VF and the sensorimotor processing took less time in the right VF, i.e., shorter RTs. A strongly supportive evidence for this assumption is that the serious hemispatial neglect more frequently occurs after lesions of the right hemisphere but not after lesions of the left hemisphere (Beis et al., 2004; Ringman et al., 2004; Becker and Karnath, 2007; Kleinman et al., 2007).

\section{SSA Deviates to the Left Side When the Eyes Fixate Straight Ahead}

It is well-known that the eye position strongly affects the perception of visual egocentric information (Barbeito and Simpson, 1991; Sridhar and Bedell, 2011, 2012). However, most previous studies did not monitor the eye position during experiments, thus the effect of eye position on visual egocentric judgment was ignored. The importance of eye position on visual egocentric judgment is caused by the fact that the retinal visual input combines with the eye position signal to build a head-centered reference frame. At the same time, the retinotopic position of an object is also transformed into the head-centered reference frame (Andersen et al., 1997). Indeed, the effect of eye position on the judgment of SSA in normal subjects has been found in previous studies (Morgan, 1978; Jeannerod and Biguer, 1989; Richard et al., 2005).

To exclude the effect of eye position on the judgment of SSA, we asked subjects to keep fixating straight ahead
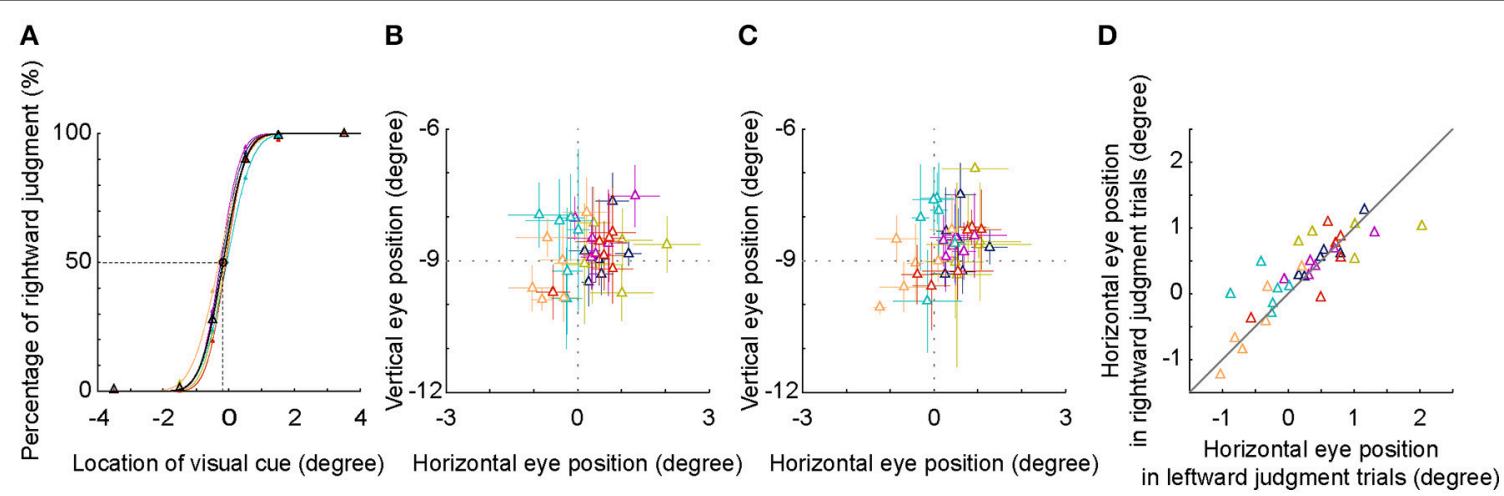

FIGURE 5 | SSA and eye position analysis. (A) The psychometric curve of each individual subject (different colors) and the averaged psychometric curve of population data (black). The PSEs of all subjects shifted to the left side of the body mid-sagittal plane, with a population PSE: $-0.18^{\circ} \pm 0.10^{\circ}$ (mean \pm SD). (B,C) Averaged eye position during an interval between visual cue onset and $250 \mathrm{~ms}$ later. Visual cue was judged as in the left side (B) or as in the right side (C). (D) Comparison of horizontal eye positions between trials with leftward and rightward judgments. Each symbol represents the data of one session. Same colored symbols represent the data of one individual subject. 
during the experiments and monitored the subjects' eye position. We found that the horizontal eye positions between the trials with opposite spatial judgments were not significantly different. Thus, the leftward deviation of SSA in the present study was not caused by the deviation of eye position.

\section{The Importance of Measuring the Location of SSA in Study of Egocentric Representation}

The location of SSA is frequently measured in the studies of egocentric representation in healthy subjects and brain damage patients. SSA reflects the location of the subjective mid-sagittal plane, which subjectively separates the egocentric space into left and right halves. Thus, the comparison between the location of SSA and body mid-sagittal plane can provide useful information to help to understand the contribution of the two hemispheres to egocentric information processing. Based on the findings from previous studies, many factors can cause the deviation of SSA, which may share different neural mechanisms. First, the SSA will deviate toward the ipsilateral side of sensory stimulation if it is presented only in one side, including neck proprioception stimulation (Karnath et al., 1994, 2002), vestibular stimulation (Karnath et al., 1994) and acute experimental painful stimulation (Bouffard et al., 2013). The deviations of SSA under these conditions are mainly due to the post-training effect, which is very different from the mechanisms leading to the deviation of SSA in our present study. Second, patients with lateralized lesion of the peripheral nervous system or visual cortices also show the deviations of SSA, such as unilateral pathologic pain (Sumitani et al., 2007; Reinersmann et al., 2012), left vestibular loss (Saj et al., 2013) and homonymous hemianopia (Ferber and Karnath, 1999; Rousseaux et al., 2013) or quadrantanopia (Kuhn et al., 2010). Third, damage in cortical regions, in particular the parietal cortex, results in the symptom of hemispatial neglect and the ipsilesional deviation of SSA (Karnath, 1994; Farne et al., 1998; Ferber and Karnath, 1999; Schindler and Kerkhoff, 2004; Richard et al., 2004a,b, 2005; Saj et al., 2006; Rousseaux et al., 2013). These findings from clinical studies

\section{REFERENCES}

Andersen, R. A., Asanuma, C., Essick, G., and Siegel, R. M. (1990). Corticocortical connections of anatomically and physiologically defined subdivisions within the inferior parietal lobule. J. Comp. Neurol. 296, 65-113. doi: 10.1002/cne.902960106

Andersen, R. A., Snyder, L. H., Bradley, D. C., and Xing, J. (1997). Multimodal representation of space in the posterior parietal cortex and its use in planning movements. Annu. Rev. Neurosci. 20, 303-330. doi: 10.1146/annurev.neuro.20.1.303

Barbeito, R., and Simpson, T. L. (1991). The relationship between eye position and egocentric visual direction. Percept. Psychophys. 50, 373-382. doi: 10.3758/BF03212230

Becker, E., and Karnath, H. O. (2007). Incidence of visual extinction after left versus right hemisphere stroke. Stroke 38, 3172-3174. doi: 10.1161/STROKEAHA.107.489096 indicate that the unbalanced egocentric information processing between the two hemispheres causes the deviation of SSA. Here we report the leftward deviation of SSA in healthy human subjects when they fixate straight ahead, which might also reflect the asymmetric process of egocentric information between the two hemispheres.

\section{CONCLUSION}

In the present study, we found that when the eyes fixated straight ahead, the visual SSA of healthy human subjects deviated to the left VF and the manual RT was longer in the left VF than in the right VF. Such results suggest that the egocentric information is asymmetrically processed between the two hemispheres.

\section{AUTHOR CONTRIBUTIONS}

YZ, MZ, and YP designed the experiments; YZ and GW collected the data; YZ and BL analyzed the data; YZ prepared all figures; YZ, MZ, and YP wrote and revised the manuscript; $M Z$ and $Y P$ supervised the experiments.

\section{FUNDING}

This study was supported by the open research fund of State Key Laboratory of Neuroscience, Shanghai Institutes for Biological Sciences, Chinese Academy of Sciences (SKLN2010A05 and SKLN-201203), and of State Key Laboratory of Cognitive Neuroscience and Learning, Beijing Normal University (CNLZD1303).

\section{ACKNOWLEDGMENTS}

We thank Dr. Yang Zhou (State Key Laboratory of Cognitive Neuroscience and Learning, Beijing Normal University) for his helpful suggestions during the experiments.
Beis, J. M., Keller, C., Morin, N., Bartolomeo, P., Bernati, T., Chokron, S., et al. (2004). Right spatial neglect after left hemisphere stroke: qualitative and quantitative study. Neurology 63, 1600-1605. doi: 10.1212/01.WNL.0000142967.60579.32

Ben Hamed, S., Duhamel, J. R., Bremmer, F., and Graf, W. (2001). Representation of the visual field in the lateral intraparietal area of macaque monkeys: a quantitative receptive field analysis. Exp. Brain Res. 140, 127-144. doi: $10.1007 / \mathrm{s} 002210100785$

Boccia, M., Nemmi, F., and Guariglia, C. (2014). Neuropsychology of environmental navigation in humans: review and meta-analysis of FMRI studies in healthy participants. Neuropsychol. Rev. 24, 236-251. doi: 10.1007/s11065-014-9247-8

Bouffard, J., Gagne, M., and Mercier, C. (2013). Effect of painful and non-painful sensorimotor manipulations on subjective body midline. Front. Hum. Neurosci. 7:77. doi: 10.3389/fnhum.2013. 00077 
Boulinguez, P., Nougier, V., and Velay, J. L. (2001). Manual asymmetries in reaching movement control. I: study of right-handers. Cortex 37, 101-122. doi: 10.1016/S0010-9452(08)70561-6

Bridgeman, B., and Graziano, J. A. (1989). Effect of context and efference copy on visual straight ahead. Vision Res. 29, 1729-1736. doi: 10.1016/0042-6989(89)90155-7

Burgess, N. (2006). Spatial memory: how egocentric and allocentric combine. Trends Cogn. Sci. 10, 551-557. doi: 10.1016/j.tics.2006.10.005

Chen, Q., Weidner, R., Weiss, P. H., Marshall, J. C., and Fink, G. R. (2012). Neural interaction between spatial domain and spatial reference frame in parietaloccipital junction. J. Cogn. Neurosci. 24, 2223-2236. doi: 10.1162/jocn_a_00260

Chokron, S., and Imbert, M. (1995). Variations of the egocentric reference among normal subjects and a patient with unilateral neglect. Neuropsychologia 33, 703-711. doi: 10.1016/0028-3932(95)00007-P

Farne, A., Ponti, F., and Ladavas, E. (1998). In search of biased egocentric reference frames in neglect. Neuropsychologia 36, 611-623. doi: 10.1016/S0028-3932(97)00164-4

Ferber, S., and Karnath, H. O. (1999). Parietal and occipital lobe contributions to perception of straight ahead orientation. J. Neurol. Neurosurg. Psychiatr. 67, 572-578. doi: 10.1136/jnnp.67.5.572

Filimon, F. (2015). Are all spatial reference frames egocentric?reinterpreting evidence for allocentric, object-centered, or world-centered reference frames. Front. Hum. Neurosci. 9:648. doi: 10.3389/fnhum.2015. 00648

Galati, G., Committeri, G., Sanes, J. N., and Pizzamiglio, L. (2001). Spatial coding of visual and somatic sensory information in body-centred coordinates. Eur. J. Neurosci. 14, 737-746. doi: 10.1046/j.0953-816x.2001. 01674.x

Galati, G., Lobel, E., Vallar, G., Berthoz, A., Pizzamiglio, L., and Le Bihan, D. (2000). The neural basis of egocentric and allocentric coding of space in humans: a functional magnetic resonance study. Exp. Brain Res. 133, 156-164. doi: $10.1007 /$ s002210000375

Goodale, M. A., and Milner, A. D. (1992). Separate visual pathways for perception and action. Trends Neurosci. 15, 20-25. doi: 10.1016/0166-2236(92)90344-8

Gross, C. G., Bender, D. B., and Rocha-Miranda, C. E. (1969). Visual receptive fields of neurons in inferotemporal cortex of the monkey. Science 166, 1303-1306. doi: $10.1126 /$ science.166.3910.1303

Gross, C. G., Rocha-Miranda, C. E., and Bender, D. B. (1972). Visual properties of neurons in inferotemporal cortex of the macaque. J. Neurophysiol. 35, 96-111.

Heilman, K. M., Bowers, D., and Watson, R. T. (1983). Performance on hemispatial pointing task by patients with neglect syndrome. Neurology 33, 661-664. doi: 10.1212/WNL.33.5.661

Jeannerod, M., and Biguer, B. (1989). [Egocentric reference and represented space]. Rev. Neurol. (Paris) 145, 635-639.

Karnath, H. O. (1994). Subjective body orientation in neglect and the interactive contribution of neck muscle proprioception and vestibular stimulation. Brain 117(Pt 5), 1001-1012. doi: 10.1093/brain/117. 5.1001

Karnath, H. O., Reich, E., Rorden, C., Fetter, M., and Driver, J. (2002). The perception of body orientation after neck-proprioceptive stimulation. Effects of time and of visual cueing. Exp. Brain Res. 143, 350-358. doi: 10.1007/s00221-001-0996-2

Karnath, H. O., Sievering, D., and Fetter, M. (1994). The interactive contribution of neck muscle proprioception and vestibular stimulation to subjective "straight ahead" orientation in man. Exp. Brain Res. 101, 140-146. doi: 10.1007/BF00243223

Kleinman, J. T., Newhart, M., Davis, C., Heidler-Gary, J., Gottesman, R. F., and Hillis, A. E. (2007). Right hemispatial neglect: frequency and characterization following acute left hemisphere stroke. Brain Cogn. 64, 50-59. doi: 10.1016/j.bandc.2006.10.005

Kuhn, C., Heywood, C. A., and Kerkhoff, G. (2010). Oblique spatial shifts of subjective visual straight ahead orientation in quadrantic visual field defects. Neuropsychologia 48, 3205-3210. doi: 10.1016/j.neuropsychologia.2010. 06.035

Land, M. F. (2012). The operation of the visual system in relation to action. Curr. Biol. 22, R811-R817. doi: 10.1016/j.cub.2012.06.049

McCourt, M. E., Mark, V. W., Radonovich, K. J., Willison, S. K., and Freeman, P. (1997). The effects of gender, menstrual phase and practice on the perceived location of the midsagittal plane. Neuropsychologia 35, 717-724. doi: 10.1016/S0028-3932(96)00115-7

Miles, W. R. (1930). Ocular dominance in human adults. J. Gen. Psychol. 3, 412-430. doi: 10.1080/00221309.1930.9918218

Milner, A. D., and Goodale, M. A. (2008). Two visual systems re-viewed. Neuropsychologia 46, 774-785. doi: 10.1016/j.neuropsychologia.2007.10.005

Morgan, C. L. (1978). Constancy of egocentric visual direction. Percept. Psychophys. 23, 61-68. doi: 10.3758/BF03214296

Neggers, S. F., Van der Lubbe, R. H., Ramsey, N. F., and Postma, A. (2006). Interactions between ego- and allocentric neuronal representations of space. Neuroimage 31, 320-331. doi: 10.1016/j.neuroimage.2005.12.028

Olson, C. R. (2003). Brain representation of object-centered space in monkeys and humans. Annu. Rev. Neurosci. 26, 331-354. doi: 10.1146/annurev.neuro.26.041002.131405

Raiguel, S., Van Hulle, M. M., Xiao, D. K., Marcar, V. L., Lagae, L., and Orban, G. A. (1997). Size and shape of receptive fields in the medial superior temporal area (MST) of the macaque. Neuroreport 8, 2803-2808. doi: 10.1097/00001756-199708180-00030

Reinersmann, A., Landwehrt, J., Krumova, E. K., Ocklenburg, S., Gunturkun, O., and Maier, C. (2012). Impaired spatial body representation in complex regional pain syndrome type 1 (CRPS I). Pain 153, 2174-2181. doi: 10.1016/j.pain.2012.05.025

Richard, C., Honore, J., Bernati, T., and Rousseaux, M. (2004a). Straight-ahead pointing correlates with long-line bisection in neglect patients. Cortex 40, 75-83. doi: 10.1016/S0010-9452(08)70921-3

Richard, C., Rousseaux, M., and Honore, J. (2005). The egocentric reference deviation of neglect patients is influenced by visuospatial attention. Neuropsychologia 43, 1784-1791. doi: 10.1016/j.neuropsychologia.2005. 02.003

Richard, C., Rousseaux, M., Saj, A., and Honore, J. (2004b). Straight ahead in spatial neglect: evidence that space is shifted, not rotated. Neurology 63, 2136-2138. doi: 10.1212/01.WNL.0000145664.09078.83

Ringman, J. M., Saver, J. L., Woolson, R. F., Clarke, W. R., and Adams, H. P. (2004). Frequency, risk factors, anatomy, and course of unilateral neglect in an acute stroke cohort. Neurology 63, 468-474. doi: 10.1212/01.WNL.0000133011.10689.CE

Rousseaux, M., Honore, J., Vuilleumier, P., and Saj, A. (2013). Neuroanatomy of space, body, and posture perception in patients with right hemisphere stroke. Neurology 81, 1291-1297. doi: 10.1212/WNL.0b013e3182a823a7

Saj, A., Cojan, Y., Musel, B., Honore, J., Borel, L., and Vuilleumier, P. (2014). Functional neuro-anatomy of egocentric versus allocentric space representation. Neurophysiol. Clin. 44, 33-40. doi: 10.1016/j.neucli.2013.10.135

Saj, A., Honore, J., Bernard-Demanze, L., Deveze, A., Magnan, J., and Borel, L. (2013). Where is straight ahead to a patient with unilateral vestibular loss? Cortex 49, 1219-1228. doi: 10.1016/j.cortex.2012.05.019

Saj, A., Honore, J., Richard, C., Coello, Y., Bernati, T., and Rousseaux, M. (2006). Where is the "straight ahead" in spatial neglect? Neurology 67, 1500-1503. doi: 10.1212/01.wnl.0000239823.28570.87

Schindler, I., and Kerkhoff, G. (2004). Convergent and divergent effects of neck proprioceptive and visual motion stimulation on visual space processing in neglect. Neuropsychologia 42, 1149-1155. doi: 10.1016/j.neuropsychologia.2004.02.006

Sheremata, S. L., Bettencourt, K. C., and Somers, D. C. (2010). Hemispheric asymmetry in visuotopic posterior parietal cortex emerges with visual short-term memory load. J. Neurosci. 30, 12581-12588. doi: 10.1523/JNEUROSCI.2689-10.2010

Sheremata, S. L., and Silver, M. A. (2015). Hemisphere-dependent attentional modulation of human parietal visual field representations. J. Neurosci. 35, 508-517. doi: 10.1523/JNEUROSCI.2378-14.2015

Sridhar, D., and Bedell, H. E. (2011). Relative contributions of the two eyes to perceived egocentric visual direction in normal binocular vision. Vision Res. 51, 1075-1085. doi: 10.1016/j.visres.2011.02.023

Sridhar, D., and Bedell, H. E. (2012). Binocular retinal image differences influence eye-position signals for perceived visual direction. Vision Res. 62, 220-227. doi: 10.1016/j.visres.2012.04.011

Sumitani, M., Shibata, M., Iwakura, T., Matsuda, Y., Sakaue, G., Inoue, T., et al. (2007). Pathologic pain distorts visuospatial perception. Neurology 68, 152-154 doi: 10.1212/01.wnl.0000250335.56958.f0 
Tootell, R. B., Silverman, M. S., Switkes, E., and De Valois, R. L. (1982). Deoxyglucose analysis of retinotopic organization in primate striate cortex. Science 218, 902-904. doi: 10.1126/science.7134981

Vallar, G., Lobel, E., Galati, G., Berthoz, A., Pizzamiglio, L., and Le Bihan, D. (1999). A fronto-parietal system for computing the egocentric spatial frame of reference in humans. Exp. Brain Res. 124, 281-286. doi: 10.1007/s0022100 50624

Zhou, Y., Liu, Y., Zhang, W., and Zhang, M. (2012). Asymmetric influence of egocentric representation onto allocentric perception. J. Neurosci. 32, 8354-8360. doi: 10.1523/jneurosci.0829-12.2012
Conflict of Interest Statement: The authors declare that the research was conducted in the absence of any commercial or financial relationships that could be construed as a potential conflict of interest.

Copyright (c) 2017 Zhou, Li, Wang, Zhang and Pan. This is an open-access article distributed under the terms of the Creative Commons Attribution License (CC BY). The use, distribution or reproduction in other forums is permitted, provided the original author(s) or licensor are credited and that the original publication in this journal is cited, in accordance with accepted academic practice. No use, distribution or reproduction is permitted which does not comply with these terms. 